\title{
Model for economic optimization and economic assessment of energy saving
}

\author{
Namitulina Anzhela Zahitovna ${ }^{1, *}$, Gorlov Viktor Vladimirovich ${ }^{2}$, Soklakova Irina Vladimirovna ${ }^{3}$, Natocheeva Natalia \\ Nikolaevna $^{4}$,Azhmuratova Madina Akhmambetovna ${ }^{5}$ \\ ${ }^{1}$ Financial University under the Government of the Russian Federation, Moscow, 125468, Russian Federation \\ ${ }^{2}$ Russian State University of Oil and Gas named I.M. Gubkin, Moscow, 119991, Russian Federation \\ ${ }^{2}$ Moscow State University of Management Moscow Government, Moscow, 107045, Russian Federation \\ ${ }^{3}$ Academy of management and production, Moscow, 123007, Russian Federation \\ ${ }^{4}$ Plekhanov Russian University of Economics, Moscow, 117997, Russian Federation \\ ${ }^{5}$ Financial University under the Government of the Russian Federation, Moscow, 125468, Russian Federation
}

\begin{abstract}
The current energy scenario should be examined from a global perspective and include models for optimizing the development and development of major renewable energy sources. The complex structural elements of such models in terms of the structure of energy consumption should include economic and environmental benefits, as well as minimize costs and negative consequences. At the current crisis stage, access to energy sources is an important asset for economic progress and world development of all states. The depletion of natural resources, ecosystem and climate change lead to the need for scientific research in the field of sustainable development and the use of various models of energy conservation to achieve social and economic growth. Renewable energy sources such as solar, geothermal, wind and others are the basis for the development of new concepts and models for economic optimization and renewable energy. The key role in the implementation of such models is played by maintaining the ecological balance and renewing energy using natural and natural resources.
\end{abstract}

\section{1 introduction}

Clean, efficient, affordable and reliable energy services are essential for global prosperity. In today's crisis and weakened by the pandemic, the economy needs to expand access to reliable and modern energy services. Poor countries strive to reduce poverty and improve the health of their citizens, for this they need to use all natural factors, solar energy and hydropower. Developed countries need additional energy resources and a reduction in energy consumption, as well as an improvement in the environmental situation. The main problems in energy consumption arise in rural areas, where most of the settlements do not have available electricity, therefore, in order to solve this problem, it is necessary to develop a model of economical energy consumption and its economic optimization.

To adapt the model of economic optimization of energy consumption, it is necessary to carry out a qualitative and quantitative analysis, as well as evaluate various approaches for the reproduction and replenishment of energy, including solar energy for cogeneration of heat and electricity.

To begin with, we will consider primary energy sources and study their features within the framework of an integrated dynamic cost optimization model. Then, at the second stage, we will consider the feasibility study of the presented model.
Then a description of the design stages is given, the development gives complete information about the functional modules. Practical experience in stimulating consumers of energy resources, as well as the development of methodological, scientific, technical and financial conditions that will increase the energy efficiency of the economic system and its optimization, which requires the involvement of methods of management theory and system analysis. Energy cost rises globally due to scarcity of fuel accompanied by increase in demand which have led to increase in wholesale prices. Additionally enormous infrastructure investments are not carried out in an endeavor to accommodate environmental and climate change policies [1]. This research seeks to realize proactive and effective framework to more energy efficiency in the manufacturing industry. This end, this research aims to promote the energy efficiency of current virtual engineering. This is centered on better integration energy prediction and optimization methods, and energy monitoring and management techniques.

\section{Methods analysis and assumptions}

This study seeks to develop a systematic procedure with the capability to inter-relate complex datasets derived from multi-dimensional factors. A logical grouping of

\footnotetext{
* Corresponding author: z.anzhela@,bk.ru
} 
captured data was organized in a broad classification of functions, data and their relationships.

Even if a energy consumption is modeled, it is still difficult to know whether what has been modeled design phase is what will be consumed at the operation phase, as these are currently non-coupled activities.

Thus, linking design parameters to energy requirements will enable to choose the most energy efficient operation, which is strongly needed in a readily applicable form.

It has been estimated that process and production optimization with respect to energy consumption could save $15-20 \%$, with another $16 \%$ savings expected to be attained by logistics optimization [2].

These savings can be achieved by redesigning process plans, redesigning machine hardware and software, replacing machine parts by more efficient ones, replacing auxiliary parts with more efficient alternatives. Today's technologies offer a wide range of developments; such as component based approach, virtual engineering technology, modeling methods, energy optimization methods, and energy management techniques. These developments have potential roles to play in achieving the engineering of more sustainable manufacturing. There is a potential for such developments to be integrated together to form new sustainable manufacturing paradigms, particularly in the context of energy optimized and reusable components.

This formed the datasets for levels of analysis. However, needs to be exploited to promote the energy efficiency of these activities.

Guided by the reference values, the datasets and the breakdown of sustainable criteria into variables, different modeling procedures and methods were selected from the geospatial analysis regime. Derived from the analyses were combined socio-economic and developmental attributes.

A majority of the problems related to sustainability identified in the research area were based on environmental indicators, which apparently stemmed from socio-economic factors.

Therefore, to assess and understand these complex interactions, the study adopted an analytical regime and makes use of information that links features and phenomena at the study area to their respective locations and relationships.

Events, patterns/trend and relationships in time and space were very crucial. The specific contribution of this study lies in the design and implementation of energy efficient systems. The energy optimization model seeks to achieve this by integrating currently unrelated energy monitoring techniques and techniques for modeling and optimizing energy at component and equipment levels based on established internal model control theory, and capitalize on and expand the powerful capabilities of the technology.

\section{Model construction}

\subsection{Current practice for energy optimization}

The focus was on an investigation of spatial patterns and relationships to guide a broader understanding of spatial patterns and processes within the research area. It was envisaged that the outcome would yield empirical information to the environmental, soc o-economic, and developmental impact. Additionally, this helped to demonstrate the operationalisation of sustainable development.

The results could provide informed knowledge and understanding to guide decision-makers towards possible areas that require further intervention with development activities, or to provide effective management and environmental protection.

In this article, we have identified the recurring steps in the process of solving the problems defined in the energy saving model. Steps included: problem formulation, problem breakdown, exploratory analysis, formulation of hypotheses, performing analysis, validating model results, final reporting and submission suggestions for further intervention.

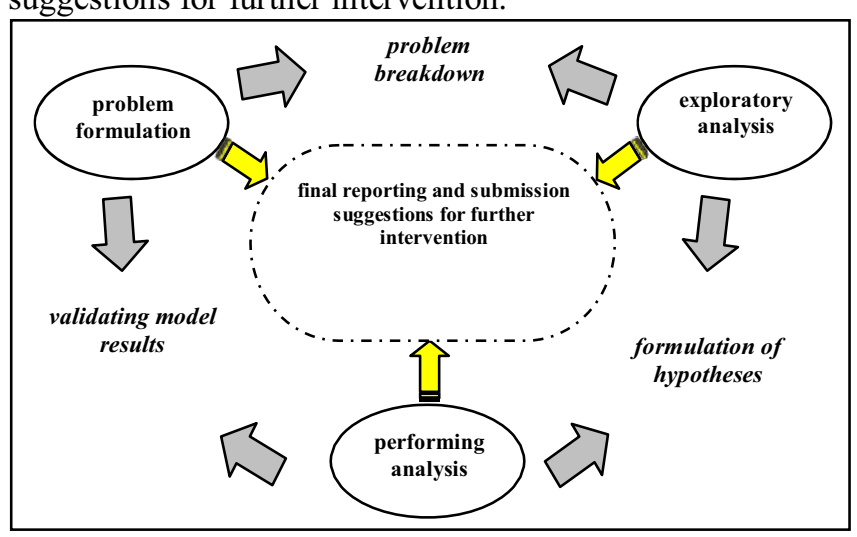

Fig. 1. Steps included.

In order to identify the opportunities for energy optimization for future manufacturing, understanding, investigating and evaluating the existing practices are perquisites. This understanding comes from monitoring, archiving and visualizing consumed the energy consumption data. The operational energy data and historical reports can be used to analyze and then identify where potential efficiency improvements are needed. Industry is using a number of methods and tools to identify the opportunities for energy optimization.

Potential energy saving opportunities can be evaluated reliably on the basis of the energy consumption reports.

\subsection{Energy consumption modeling}

Considerable efforts have been invested by many researchers in order to model or predict the energy usage. The energy consumption modeling can be classified into three main methods. The energy modeling methods are:

1. thermal modeling

2. mathematical modeling

3. state-transition modeling. 
The use of methods is not common in spatial analysis, but it is increasingly used in the environmental modeling and even economic indicators.

This is methods begin with a recognition that the spatial variation of any continuous attribute is often too irregular to be modeled using a simple, smooth mathematical function.

The primary tool in analysis is a variogram that displays the variances within groups of observations, which are plotted as a function of the distances between observations.

This method determines whether data exhibit spatial dependencies, i.e., measurements at points that are close together are more similar than those that are further apart. The variogram consists of an experimental variogram calculated from the data and a variogram model, or theoretical variogram, which is fitted to the data.

This is defined as follows:

$$
2 \gamma(h)=E\{[Z(u)-Z(u+h)]\} 2
$$

where:

$-2 \gamma(\mathrm{h})$ represents the variogram at $\mathrm{h}$

- $\mathrm{h}$ represents the distance $=u \mathrm{u}-\mathrm{uj}$, ( lag)

- $\mathrm{u}$ (location vector) represents all possible locations

- $Z$ represents the data

- E represents mean.

Mathematical modeling aims to optimize the energy consumption of a system by define it as a function of its working parameters. Collected energy data mathematically, and finally the energy data were linked to the process. Mathematical modeling can give good results in term of reducing energy consumption, it does not consider any physics involved to ease the modeling process. Since this method formulates the consumed energy behavior of the whole operation by considering the energy consumption at each individual operating state or event, it is considered to be generic, scalable to the system size and details required, considers how the system is being used, and needs small computational capabilities [3].

\subsection{Energy consumption modeling}

From Figure 2, the built System represents the energy consumption is to be monitored.

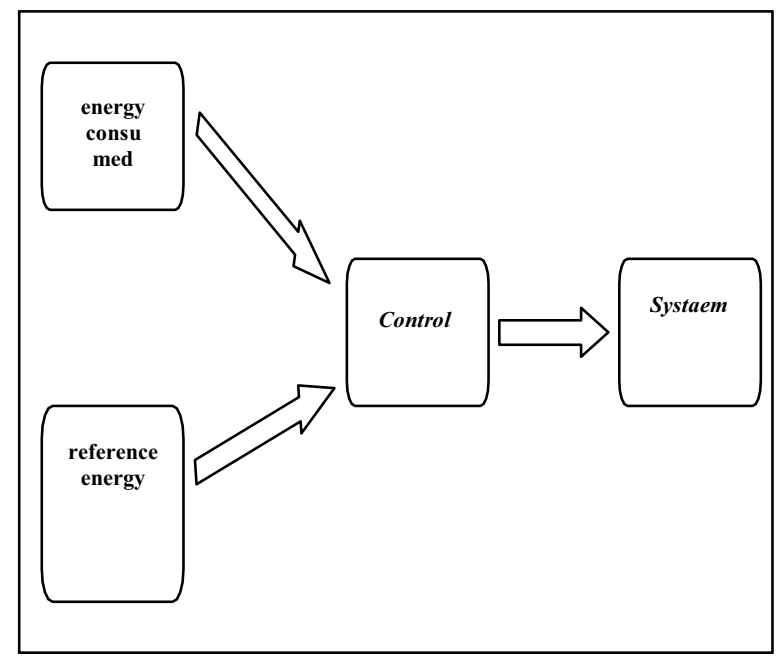

Fig. 2. Basic feedback theory

$\boldsymbol{U}$ - is the energy consumed by the System at its operation phase, and it is monitored. $\boldsymbol{P}$ - is reference energy consumption.

The Control (decision maker or software) is fed by $\mathrm{P}$ and $\mathrm{U}$, and according to some rules and restrictions it provides the actuating action to operate the System accordingly. The blocks shown in of figure 2 represent the sub-systems that form the control process of a manufacturing system energy consumption, and the connected arrows represent the transformed variables. $\mathrm{R}$ (d) is an unknown disturbance that could affect System, hence its energy consumption.

It could be internal disturbance or external disturbance such as excessive environmental conditions or unexpected increase of work load.

$\mathrm{U}$ is the action taken by the Control to make System performs its functions yet consumes the optimized amount of energy[4].

$\mathrm{U}$ is introduced to both the System and its System Model$^{\wedge}$.

$\mathrm{Y}$ is the actual energy consumption by System, which is then to be compared with the predicted energy of System $\mathrm{Model}^{\wedge}$, resulting in $\mathrm{R}(\mathrm{d})^{*}$ as a difference between the actual and predicted energy consumption.

$$
R^{*}=\left(F-F^{*}\right) Y+R
$$

That is If $\mathrm{F}=\mathrm{F}^{*}$ which can be achieved by exact modeling, then $\mathrm{R}^{*}$ is equal to the unknown disturbance R.

On the other hand, if the System F works in normal operating conditions and no presence of any disturbance is observed, then $\mathrm{R}$ becomes zero, meaning $\mathrm{R}^{*}$ is a measure of energy consumption discrepancies between the System F and its System Model^ ${ }^{\wedge}$.

Thus, $\mathrm{R}^{*}$ may be considered as the energy information that is missing in the virtual System Model $\mathrm{F}^{*}$, and can therefore be used to improve controlling the actual energy to be consumed by the real System F.

This can be done by subtracting $\mathrm{R}^{*}$ from reference energy threshold J.

$$
Y=\left(J-R^{*}\right) C=\left(J-\left(F-F^{*}\right) Y-R\right) C
$$

It is worth noting that, even if $\mathrm{F} \neq \mathrm{F}^{*}$, perfect disturbance $\mathrm{d}$ eliminating can still be achieved provided $\mathrm{C}=1 / \mathrm{G}^{*}$.

\section{Empirical results and analysis}

Only one component is considered by each experiment, since the interest of this research focuses on componentbased domain, and how the component configuration affects its energy consumption as well as the whole energy consumption.

Also, for each experiment, only one parameter (acceleration value, component operation mode or sequence of operation) is altered whereas other parameters stay as in their original settings and configurations [5].

The equation below represents the total energy 
consumption of a modeled system depending on operating conditions (idle, operating, stand-by or off) of each one of its components:

$$
F 1=\frac{\sum_{1}^{k} a_{k} b_{k}}{k_{1}} ; \sum_{1}^{k} a_{k}=1
$$

where: F1- general criterion of assessment; $a_{k}$ weight of the k-th criterion; $b_{k}$ - the value of the dimension of the $\mathrm{k}$-th criterion; $\mathrm{K}(\mathrm{k})$ - the number of particular criteria in the ranked series.

The coefficient of determination, from the extrapolation method, is used as an additional evaluation criterion, it can take values in the range from 0 to 1 . The closer it is to one, the more stable is the process of changing the studied indicator - growth or decline. To assess the degree of trend stability, the following scale of the determination coefficient can be used: if its value is less than 0.4, then this is an unstable process (weak trend); if it is from 0.4 to 0.7 - a fairly stable process (a noticeable trend); over 0.7 is a very stable process (strong trend).

With a small length of the initial time series, the accuracy of the forecast can be improved by calculating the weighted average value of the forecasts of the studied indicator $\mathrm{Y}$ according to several models:

$$
\bar{y}_{n+k}=\frac{\sum_{j=1}^{m} \hat{y}_{n+k j} \cdot R_{j}^{2}}{\sum_{j=1}^{m} R_{j}^{2}}
$$

where is the average predicted value $\mathrm{Y} ; \mathrm{n}$ is the length of the original time series (number of the last level);

$\mathrm{k}$ - lead period (forecasting horizon); $\hat{y}_{n+k_{j}}$ - forecast by model $\mathrm{j} ; R_{j}^{2}$ - is the coefficient of determination of the model $\mathrm{j}$. In order to determine the effectiveness of the organizational process for the distribution of defense orders at the defense industry enterprises in the regions, it is necessary to apply indicators that determine all stages of the placement process: the planning stage, the placement stage, the execution control stage.

This model makes the following patristic contributions to the scientific field of energy-saving systems research:

1. A deep understanding of the current constraints that need to be addressed and the requirements that need to be met in order to propose and design an efficient framework for predicting and optimizing the energy consumption of industrial systems.

2. The energy saving system must use virtual design tools throughout the energy saving life cycle.

3. Proactive, applicable and comprehensive framework for energy prediction and optimization within the component virtual design tools, which allows the implementation of verified control configurations.

\section{Conclusion}

In this article, we have proposed an Economic Optimization Model that allows us to analyze external and internal factors affecting energy conservation based on quantitative indicators with structured qualitative characteristics

All assessed indicators are formally described by mathematical variables. Functions are constructed for fuzzy sets that characterize the semantics of the values of linguistic numbers. Based on the use of the constructed mathematical model of energy saving, a full factorial experiment was set up, the processing of the results of which made it possible to include in an analytical form the relationship between the input and output values.

The constructed model makes it possible, on a formalized basis, to take into account external and internal causative agents and negative factors of influence on energy saving, used in the process of making decisions on the optimization of energy saving, to accumulate and use them when forming an economic assessment of energy saving.

\section{References}

1. Kaufmann, A., Gupta, M.Fuzzy Mathematical Models in Engineering and Management Science. (Amsterdam: Elsevier, 1998)

2. Dombey, J., Tóth-Laufer, E.Reducing computational requirements in fuzzy control of the Mamdani type. (ActaPolytechnicaHungarica, 17(3), 2020)

3. L A. Dietmair and A. Verl, Energy consumption forecasting and optimisation for tool machines, (Energy, 62, 2009)

4. Zhou Deng, Jiang yongbiao Construction and application of 3D geological model of 3DMine in No.3 ore belt of Zoujiashan uranium deposit (Journal of geology, 41 (1), 2017)

5. "Environmental management - Life cycle assessment - Requirements and guidelines," International Organization for Standardization (2016) 